\title{
Effects of Empirical Glucocorticoid Use on Severe Acute Exacerbation of COPD During Hospitalization
}

\author{
Ling $\mathrm{Wu}^{\mathrm{l}, *}$ \\ Nan $\operatorname{Lan}^{2, *}$ \\ Xiaoyu Yang ${ }^{3}$ \\ 'Department of Respiratory Medicine, \\ The First People's Hospital of Shuangliu \\ District, Chengdu, People's Republic of \\ China; ${ }^{2}$ Department of Respiratory \\ Medicine, The Affiliated Hospital of \\ Southwest Medical University, Luzhou, \\ People's Republic of China; ${ }^{3}$ Department \\ of Respiratory Medicine, Peking \\ University First Hospital, Beijing, People's \\ Republic of China
}

*These authors contributed equally to this work
Correspondence: Xiaoyu Yang

Email 18IIII0229@pku.edu.cn.com
Background: Clinicians' selection of glucocorticoids during hospitalization of COPD patients is often based on the medical staff's judgment of the patient's condition, and there is no objective judgment standard. The purpose of this study was to investigate the outcome of severe COPD deterioration in patients treated with glucocorticoid and without glucocorticoid during hospitalization.

Methods: This study was an observational cohort study. Data on hospitalization with severe COPD deterioration were collected and followed up for 1 year. One year after discharge, the re-hospitalization due to COPD was collected retrospectively. The patients were divided into glucocorticoid group and control group according to whether the patients were given glucocorticoid therapy or not when they were admitted to hospital for the first time. The primary outcome was rate of future COPD exacerbations, while the secondary outcome was hospital stay, treatment cost and COPD-related readmission time. These results are analyzed by using Poisson model and Cox regression model.

Results: A total of 91 patients were enrolled in the study, including 39 in the control group and 52 in the glucocorticoid group. The annual rate of future COPD exacerbations in the glucocorticoid group was significantly lower than that in the control group (RR,0.50 [95\% CI, 0.26-0.98]; $\mathrm{P}=0.045)$. The risk of COPD recurrence in the glucocorticoid group was lower than that in the control group, as assessed in a time-to-first-event analysis (HR, 0.46 [95\% CI 0.22-0.97]; $\mathrm{P}=0.042$ ). Subgroup analysis found that in patients with blood eosinophil $<100$ cells $/ \mu 1$, the future annual severe exacerbation rate of glucocorticoid group was significantly lower than that in the control group (adjusted RR,0.37 [95\% CI 0.17-0.83]; P = 0.016).

Conclusion: The use of glucocorticoids during hospitalization in COPD can more effectively reduce the severe deterioration of COPD than without glucocorticoids.

Keywords: COPD, glucocorticoid, exacerbation

\section{Introduction}

Chronic obstructive pulmonary disease (COPD) is a common respiratory disease characterized by persistent airflow limitation. The primary clinical symptoms are cough, expectoration and dyspnea, and the morbidity and mortality are gradually increasing. The latest research by Wang et al shows that in China, the overall prevalence rate of COPD is $8.6 \%$. The prevalence rate of COPD in people over 40 years old is $13.7 \%$. About 25 million people worldwide die of chronic obstructive pulmonary disease (COPD) every year. ${ }^{2}$ COPD ranks fourth in the cause of death in the world and seventh in the burden of disease. ${ }^{3,4}$ 
Acute exacerbation of COPD refers to the aggravation of respiratory symptoms, the degree of change is beyond the range of daily variation and leads to changes in drug treatment. The therapeutic drugs for COPD primary include bronchodilators, glucocorticoids, antibiotics, expectorants and so on. In stable COPD patients with FEV1 $<60 \%$ predicted, regular inhalation of glucocorticoids can improve symptoms, lung function, improve quality of life and reduce the frequency of acute exacerbation. Clinical studies have also shown that glucocorticoid use in AECOPD patients can shorten recovery time, improve pulmonary function and hypoxemia, and reduce the risk of recurrence and treatment failure, ${ }^{5}$ but may lead to an increased risk of pneumonia and fungal infection. ${ }^{6}$ Therefore, the clinical benefits and risks of patients with COPD need to be fully considered in both acute exacerbation and stable phase. The number of eosinophils has been used as the only biological indicator of long-term inhalation of sex glucocorticoids in stable COPD, but at present, there is no unified standard in the guidelines for the selection of glucocorticoid therapy in patients with AECOPD, so the clinical selection is quite different, and the long-term effect is not exact. ${ }^{7}$ Medical staff often make empirical judgment on the glucocorticoid selection of patients with AECOPD based on the judgment of patients' symptoms.

Therefore, this study investigated the indicators and prognosis of patients with severe COPD deterioration treated with and without glucocorticoid therapy during hospitalization, to understand the clinical benefits of glucocorticoid use, and to predict the factors affecting the long-term prognosis of glucocorticoid by subgroup analysis and interaction study.

\section{Methods}

This study is a 1 year observational study. Through electronic medical records, a retrospective screening was conducted on all hospitalized patients with severe COPD who occurred in West China Airport Hospital of Sichuan University (Shuangliu District first people's Hospital) between January 1, 2017 and June 1, 2018. The primary diagnosis of hospitalization must be registered as "acute exacerbation of COPD". This study was registered in the Chinese Clinical Registry (ChiCTR2000038966). The data used in this study is obtained from the previous clinical diagnosis and treatment in our hospital, and the risk to the subjects of this study is not greater than the minimum risk. Exemption of informed consent will not adversely affect the rights and health of the subjects. The privacy and personal identification information of the subjects are protected. Therefore, the ethics committee of our hospital does not require patients to agree to review their medical records. The present study was approved by the ethics committee of the hospital and adhered to the tenets of the Declaration of Helsinki.

\section{Study Patients}

All patients aged between 18 and 95 met the diagnostic criteria for acute exacerbation of COPD in the 2018 revised Global Initiative for chronic obstructive Pulmonary Disease (GOLD) (8). There was a report of stable pulmonary function examination within 6 months before admission, and the compliance was good. ICS/ LABA or ICS/LABA+LAMA was used in the stable period. Patients with other lung diseases (such as asthma, pulmonary interstitial disease, pulmonary tuberculosis, etc.), severe chronic diseases, malignant tumors and those who were unable to provide hospitalization information due to COPD one year before and after admission were excluded.

\section{Sample Size Estimation}

According to PASS15.0 calculations, 41 cases and 41 controls are required to achieve 95\% efficacy and 5\% alpha error. We collected 39 cases of control group and 52 cases of glucocorticoid group, achieving 93\% efficacy and $5 \%$ alpha error.

\section{Data Acquisition}

The data collected manually through the hospital electronic medical record system include the following: demographic data; clinical characteristics; complications; Date, time and outcome of laboratory blood tests in the hospitalization; medical expenses and information relevant to all hospitalization records such as specific medication methods for deterministic treatment components (antibiotics/ glucocorticoids) in hospitalization, start and stop dates, by discharge records provided by the patient and the readmissions due to COPD in the hospital database, collection of readmission treatment details, readmission date. Patients were consulted by telephone about the number of acute exacerbations of COPD one year before admission. Other data manually extracted from the outpatient system include: stable lung function within 6 months before admission; the patient's outpatient medication list and medical records; and long-term maintenance 
medication components (ICS/LABA or ICS/LABA +LAMA) before and after discharge.

\section{Grouping Criteria and Research Variables}

According to the inpatient doctor's advice each eligible subject will be assigned to glucocorticoid group (glucocorticoid used) and control group (no glucocorticoid use). The primary outcome was the annual severe exacerbation rate. Severe exacerbation of COPD was defined as the annual rate of future COPD exacerbations and hospitalization in addition to systemic glucocorticoids and/or antibiotics. ${ }^{9}$ The annual severe exacerbation rate is defined as the number of serious deterioration per person per year. The secondary outcome were hospital stay, treatment cost and COPD-related readmission time and the time of readmission for the first time associated with COPD.

\section{Statistical Analysis}

Using spss23.0 statistical software to complete the statistical analysis, using bilateral test, $\mathrm{P}<0.05$ will be considered to be statistically significant (except for special instructions). If it accords with normal distribution, the comparison of continuous parameters between the two groups is calculated by $t$-test; if it conforms to non-normal distribution, the comparison of continuous parameters between the two groups is calculated by MannWhitneyU test. The frequency difference between groups passed the chi-square test.

The primary analysis is to use Poisson regression model to compare the annual severe deterioration rate of each patient, and to observe the number of days as a compensation variable. Secondary analysis uses KaplanMeier charts and Cox proportional hazard models for event analysis to estimate the first increased risk ratio.

Two subgroup analyses were performed to illustrate the potential response predictors. For the first time, a subgroup analysis was made for baseline blood eosinophil count and the number of hospitalizations due to COPD in the past 12 months before the first admission. The second analysis included the same two covariates and the correlation analysis of each variable at the baseline. The variables that had great influence on the outcome and gender, age, BMI, smoking, complications and GOLD classification were selected for subgroup analysis.

Two sensitivity analyses were carried out, and the primary outcome indicators were analyzed according to the first admission glucocorticoid group and the control group for the first time. The first sensitivity analysis only included people with the same admission and re-admission treatment groups. After the second sensitivity analysis was divided into groups according to the specific re-admission glucocorticoid therapy, the re-admission grouping method was consistent with the first admission treatment mode.

\section{Results}

A total of 898 candidate inpatients were included, including 52 patients in the glucocorticoid group, 39 patients in the control group, 42 patients in the glucocorticoid group and 33 patients in the control group for the first sensitivity analysis. The specific treatment methods were divided into systemic glucocorticoid group $(\mathrm{n}=32)$, local glucocorticoid group $(n=20)$ and control group $(n=39)$. The second sensitivity analysis included systemic glucocorticoid group $(\mathrm{n}=21)$, local glucocorticoid group $(\mathrm{n}=15)$ and control group $(\mathrm{n}=33)$ (Figure 1). Among these patients, there was no significant difference in baseline (Table 1). The control group CRP was significantly higher than the glucocorticoid group see Table E1.

\section{Primary Outcome}

In order to study the effect of glucocorticoid use on severe deterioration during hospitalization, the annual COPD severe exacerbation rate in the glucocorticoid group was compared with that in the control group, and it was found that the glucocorticoid group significantly decreased (adjusted RR,0.50 [95\% CI 0.26-0.98]; $\mathrm{P}=0.045$ ) (Figure 2). Considering that the treatment of COPD readmission may be different from that of first-admission glucocorticoid use, we analyzed the population in which the glucocorticoid use of re-admission was the same as that of first-admission glucocorticoid use, and there was no significant difference between the two groups in the first sensitivity analysis (Figure 3 ). In view of the poor stability of the appeal study, and the fact that glucocorticoid therapy during hospitalization includes systemic glucocorticoid therapy and local glucocorticoid therapy, and there may be differences between systemic glucocorticoid therapy and local glucocorticoid therapy, we are divided into groups according to patients' glucocorticoid use patterns (see Table E2 for details of glucocorticoid use during hospitalization). The study found that the serious deterioration rate of systemic glucocorticoid COPD in the control group was significantly lower than that in the control group (adjusted RR. 0.28 [95\% CI 0.12-0.68]. P $=0.005)$, there was no significant difference in the severe exacerbation rate of COPD between the local use of 


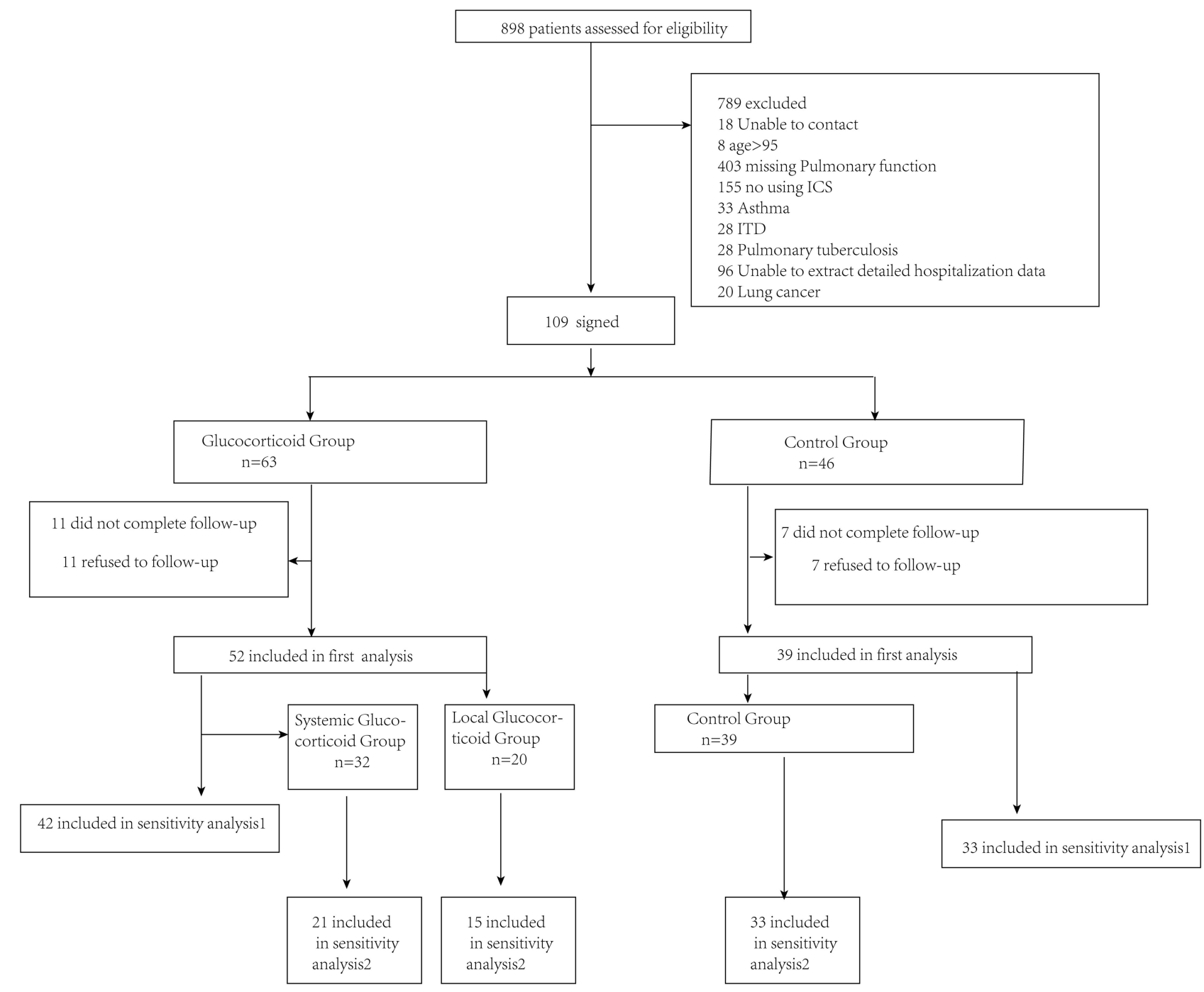

Figure I Flow chart of patient registration process.

glucocorticoids and the control group (Table E3). The second sensitivity analysis was carried out for the people whose re-admission glucocorticoid use group was the same as that of the first glucocorticoid group. Similar results were obtained, as shown in Table E4. This means that systemic glucocorticoid use during hospitalization can effectively reduce the severe deterioration of COPD in patients.

Considering that eosinophil count and the number of readmission due to COPD before admission are independent factors affecting readmission, we conducted a subgroup analysis of these two factors. GOLD guidelines take blood eosinophil $>300$ cells $/ \mu \mathrm{L}$ as a recommended indicator for long-term inhalation of glucocorticoids, while patients with blood eosinophil $<100$ cells $/ \mu \mathrm{L}$ are opposed to long-term inhalation of glucocorticoids. Since the median eosinophil count of patients we collected was 80 $(30-170)$, we took blood eosinophil $<100$ cells $/ \mu \mathrm{L}$ as the key point. In patients with blood eosinophil $<100$ cells $/ \mu \mathrm{L}$, the annual severe exacerbation rate of glucocorticoid group was significantly lower than that in the control group (adjusted RR,0.37 [95\% CI $0.17-0.83] ; \quad \mathrm{P}=$ 0.016). There was no significant difference in severe exacerbation rate between the two groups with blood eosinophil $\geq 100$ cells $/ \mu \mathrm{L}$ (Figure 4 ). This means that AECOPD patients with eosinophil $<100$ cells $/ \mu \mathrm{L}$ can still get good clinical benefits by using glucocorticoids. Based on the subgroup analysis of COPD admission before admission, it was found that there was no significant difference in the annual serious exacerbation rate of COPD between the glucocorticoid group and the control group (Figure 5). 
Table I Characteristics of the Patients at Baseline

\begin{tabular}{|c|c|c|c|c|}
\hline Parameter & Total $(\mathbf{N}=91)$ & Control Group $(n=39)$ & Glucocorticoid Group $(n=52)$ & $P$ \\
\hline Age, $y$, median (IQR) & $69(63-78)$ & $74(63-80)$ & $67(63-76)$ & 0.164 \\
\hline Men, No.(\%) & $56(61.5)$ & $23(59)$ & $33(63.5)$ & 0.663 \\
\hline $\mathrm{BMI}, \mathrm{mean} \pm \mathrm{SD}$ & $21.04 \pm 3.03$ & $20.4 I \pm 2.42$ & $21.52 \pm 3.36$ & 0.082 \\
\hline Former smoker,No.(\%) & $55(60.4)$ & $24(6 \mid .5)$ & $3 I(59.6)$ & 0.853 \\
\hline Comorbidities, yes, No./total (\%) & $70(76.9)$ & $33(84.6)$ & $37(7 \mid .2)$ & 0.131 \\
\hline Smoking pack-years, median (IQR) & $21(19-23)$ & $20(0-40)$ & $10(0-30)$ & 0.435 \\
\hline COPD treatment, No. (\%) & & & & 0.777 \\
\hline ICS/LABA & $69(75.8)$ & $29(74.4)$ & $40(76.9)$ & \\
\hline ICS/LABA/LAMA & $22(24.2)$ & $10(25.6)$ & $12(23.1)$ & \\
\hline Gold grade, No. (\%) & & & & 0.508 \\
\hline 1 & $9(9.9)$ & $2(5.1)$ & $7(13.5)$ & \\
\hline 2 & $37(40.7)$ & $17(43.6)$ & $20(38.5)$ & \\
\hline 3 & $36(39.6)$ & $17(43.6)$ & $19(36.5)$ & \\
\hline 4 & $9(9.9)$ & $3(7.7)$ & $6(11.5)$ & \\
\hline $\mathrm{FEVI} \%$ predicted, mean $\pm \mathrm{SD}$ & $52.34 \pm 19.69$ & $51.54 \pm 17.26$ & $52.94 \pm 21.48$ & 0.739 \\
\hline COPD admission in previous year & $0(0-0)$ & $0(0-0)$ & $0(0-0)$ & 0.477 \\
\hline 0 & $73(80.2)$ & $30(76.9)$ & $43(82.7)$ & 0.792 \\
\hline 1 & $12(13.2)$ & $6(15.4)$ & $6(11.5)$ & \\
\hline$\geq 2$ & $6(6.6)$ & $3(7.7)$ & $3(52)$ & \\
\hline Eosinophil count (cells per $\mu \mathrm{L}$ ), median (IQR) & $80(30-170)$ & $80(10-150)$ & $85(30-180)$ & 0.184 \\
\hline Neutrophil \%,median (IQR) & $77.1(65.7-83.5)$ & 79.3(66.7-84.8) & $75.5(64.7-83.1)$ & 0.169 \\
\hline
\end{tabular}

Note: The data are expressed as $n(\%)$, average $\pm S D$ or median [quartile interval].

Abbreviations: ICS, inhaled glucocorticoids; LABA, long-acting $\beta$ agonists; LAMA, long-acting muscarinic antagonists.

In order to study the factors affecting the relationship between glucocorticoids and severe deterioration, we studied the correlation between all variables (Figure 6). We found that the variables related to the number of readmissions after discharge were the number of hospitalizations due to COPD within one year before admission $[\mathrm{r}=$ $-0.23]$ and the number of eosinophils before first admission $[r=0.24]$. Gender, age, BMI, PO2, smoking history, complications and Gold grade were included in the interaction analysis. It was found that more clinical benefits were obtained during hospitalization in COPD patients over 65 years old, male, normal BMI, no smoking, concomitant symptoms, GOLD3-4, stable use of ICS/LABA and $\mathrm{PO}_{2} \geq 60 \mathrm{mmhg}$ during hospitalization (Figure 7).

\section{Secondary Outcome}

As shown by the Kaplan-Meier curve of the first serious deterioration time of COPD, the first severe deterioration time of the glucocorticoid group was significantly later than that of the control group $(\mathrm{p}=0.028)$. (Figure 8). Using the Cox model, the HR between the glucocorticoid group and the control group was $0.46(\mathrm{P}=0.042)$.

There was no significant difference in hospitalization time and hospitalization cost between the two groups. The median length of stay in the glucocorticoid group was 13 days (IQR, 9-15), with an average hospitalization cost of 8448.65 yuan (SD, 2292.20), while that in the control group was 11 days (IQR, 9-13), with an average cost of 7656.30 yuan (SD, 1999.26). (Figures 9 and 10)

\section{Adverse Reaction}

In the course of hospitalization, a total of 17 adverse events occurred. Hyperglycemia was found in 3 cases, gastrointestinal reaction in 13 cases and osteoporosis in 2 cases. There was no difference in the incidence of adverse events between the glucocorticoid group and the control group. Table 2 


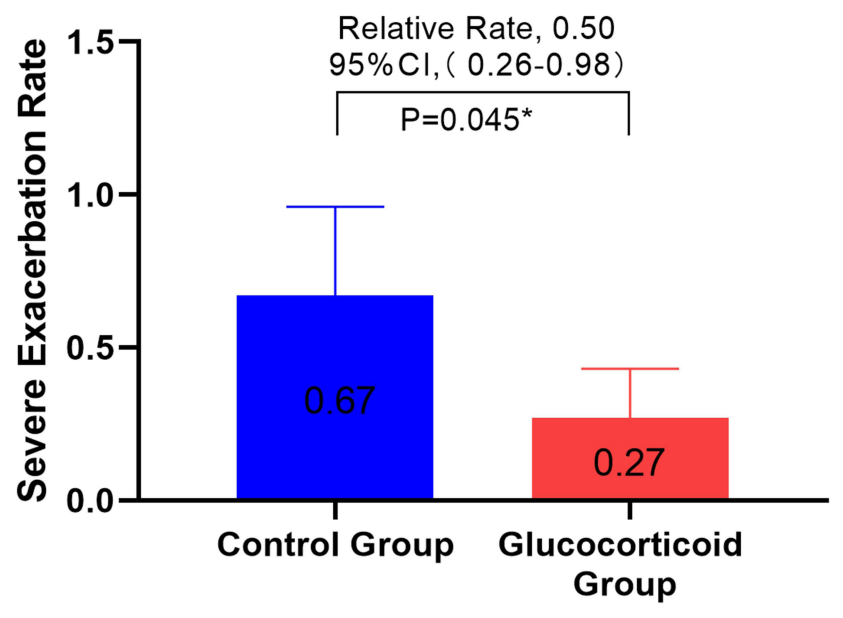

Figure 2 The ratio of the serious deterioration rate of COPD between the glucocorticoid group and the non-glucocorticoid group within one year after discharge, adjusting the number of hospitalization due to COPD in the year before admission, the number of eosinophil cells and the number of CRP before admission for the first time. The data in the bar chart represents the mean. *Means the difference was statistically significant.

Relative Rate, 0.50

$95 \% \mathrm{Cl},(0.23-1.06)$

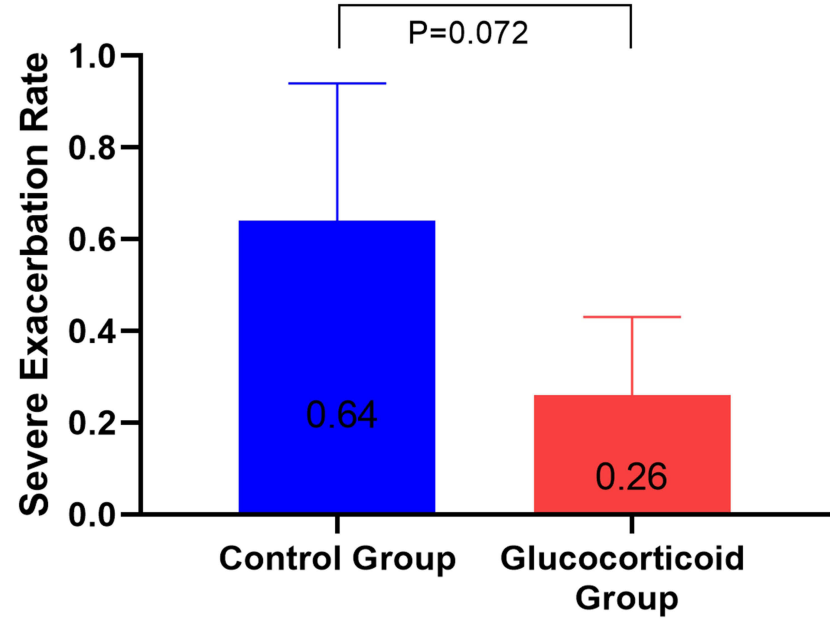

Figure 3 Only the people in the re-admission treatment group and the first admission group were analyzed. The ratio of the serious deterioration rate of COPD in the glucocorticoid group and the non-glucocorticoid group within one year after discharge was adjusted. The number of admission due to COPD, the number of blood eosinophils and the number of CRP in the first year before admission were adjusted.

\section{Discussion}

So far, there is still no uniform standard for the selection of glucocorticoids during hospitalization in COPD. Due to the side effects of glucocorticoids, medical staff and patients may be conservative in the selection of glucocorticoids. Although previous studies have shown that eosinophils can be used as a biological indicator of stable inhaled glucocorticoids, there are no studies on the long-
Control Group

Glucocorticoid Group

Relative Rate, 0.54

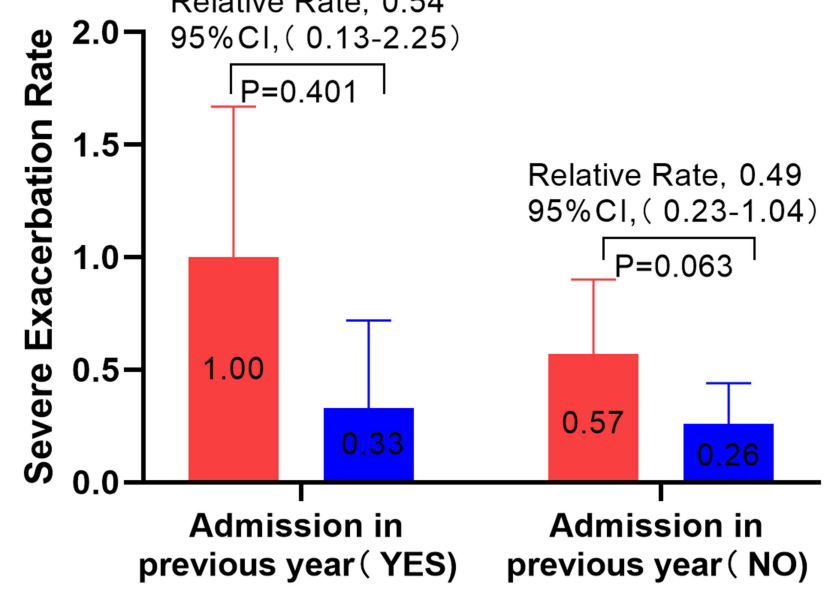

Figure 4 A subgroup analysis was made on whether there was acute aggravation of COPD one year before the first admission, and the ratio of severe deterioration of COPD between the glucocorticoid group and the non-glucocorticoid group.

term benefits and influencing factors of glucocorticoids during hospitalization.

In our study, our results show that systemic glucocorticoids during hospitalization can significantly reduce the severe deterioration rate of COPD; in the past 20 years, related studies have shown ${ }^{9-17}$ (Table 3) that glucocorticoids can significantly reduce the risk of COPD recurrence and prolong the time of recurrence, which is consistent with our results. However, more than $70 \%$ of the studies took place before 2007, when the long-term stable inhalation drugs and methods of use of COPD patients were immature, so the results at that time could not objectively reflect the current situation. In addition, the maximum follow-up time of all studies is limited to half a year, only the recurrence rate and treatment failure rate have been studied, and the design of setting the follow-up time at 6 months may come from the design principles of the SCCOPE study in $1998^{18}$ and the results of Dennis et al in 1999. ${ }^{13}$ Dennis et al designed a six-month RCT trial and found that glucocorticoids significantly reduced the rate of first treatment failure for 30 days $(23 \%$ vs $33 \%, \mathrm{P}=0.04)$ and 90 days $(37 \%$ vs $48 \%, \mathrm{P}=0.04)$ compared with placebo. There was no significant difference in the sixmonth treatment failure rate $(51 \%$ in the combined glucocorticoid group and $54 \%$ in the placebo group, $\mathrm{P}=0.58$ ). It was not until 2007 that the long-term natural history study of severe exacerbation and death of chronic obstructive pulmonary disease (COPD) revealed the rule of severe 


\section{Control Group}

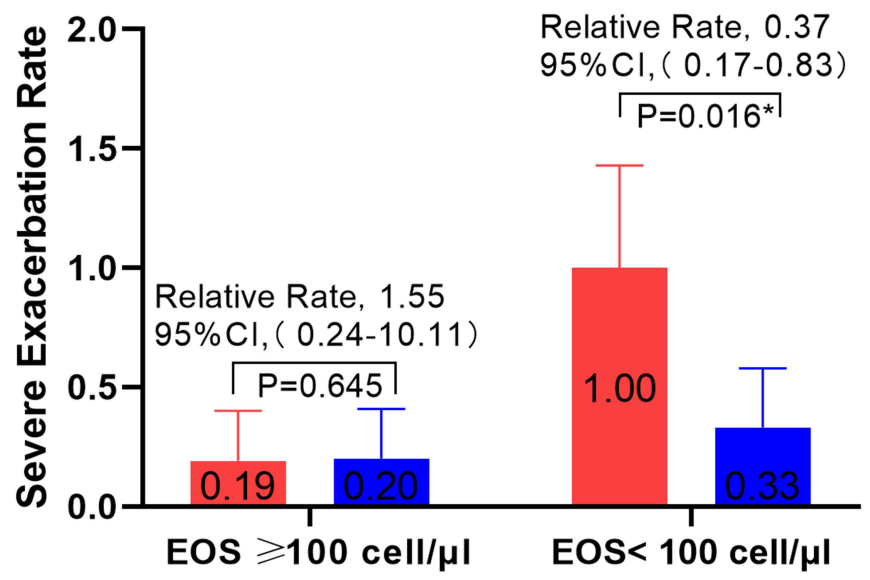

Figure $5 \mathrm{~A}$ subgroup analysis was made on whether eosinophil was less than 100 cell/ $\mu \mathrm{L}$ before admission for the first time, and the ratio of severe deterioration rate of COPD between glucocorticoid group and non-glucocorticoid group. * Means the difference was statistically significant.

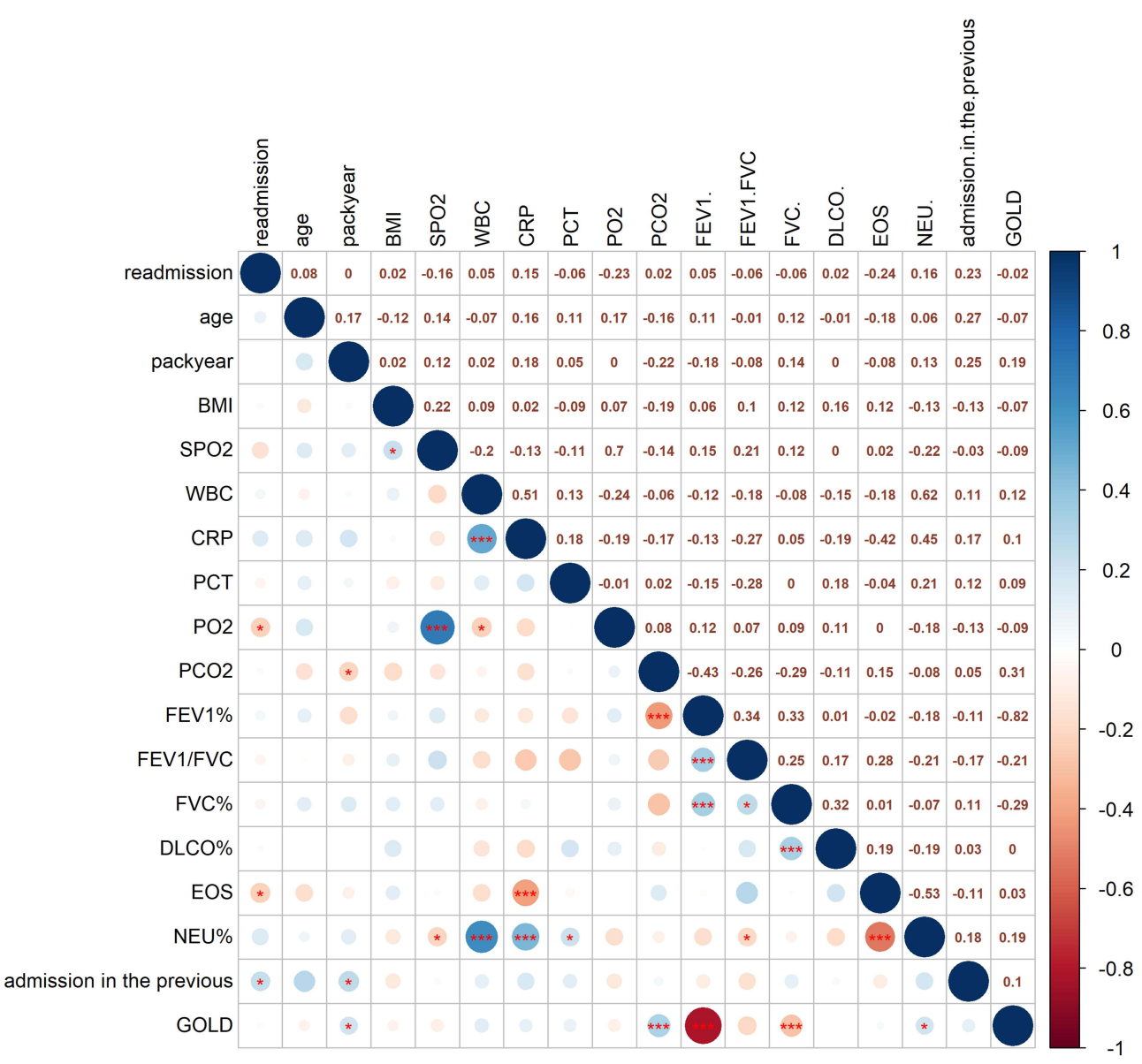

Figure 6 Bivariate correlation analysis of AECOPD patients: * means $0.05 \mathrm{~mm} \geq p>0.01$; ***means $\leq 0.00 \mathrm{I}$. The data in the box represents the $r$ value.

exacerbation of COPD.${ }^{19}$ Therefore, the researchers at that time may not realize that the change in the number of acute events each year may have an impact on the frequency of acute exacerbation thereafter, even if hospitalization a year ago may have a long-term impact on subsequent COPD, so we raised the study time to 1 year. 


\section{Favors Glucocorticoid Group}

Favors Control Group

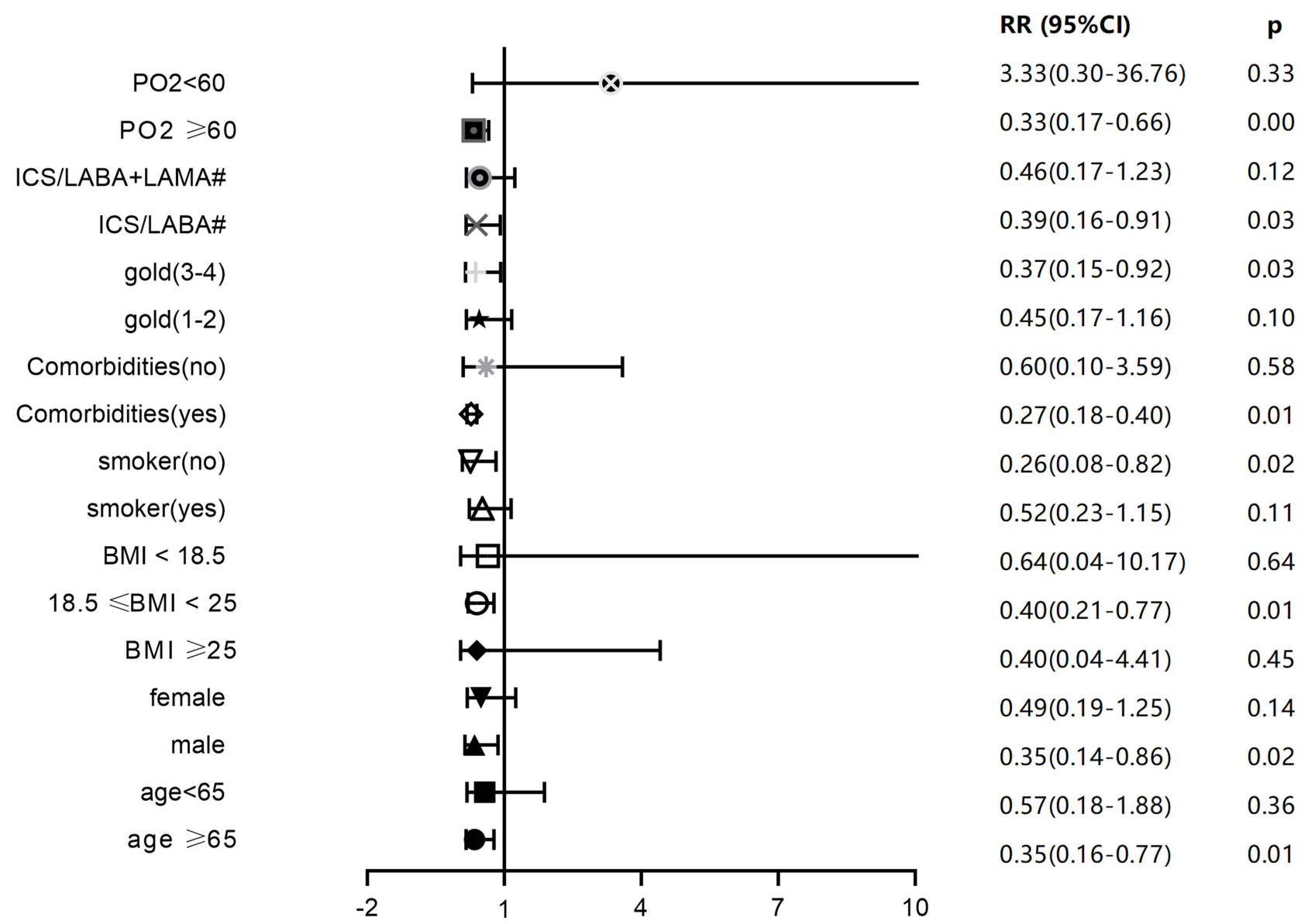

Figure 7 Ratio of severe deterioration of COPD in glucocorticoid group and non-glucocorticoid group in univariate model. BMI < I8.5 indicates malnutrition, BMI $\geq 25$ indicates overnutrition, age $>65$ represents advanced age, $\mathrm{PO}_{2}<60$ indicates hypoxemia, GOLDI-2 represents mild to moderate airflow limitation, GOLD3-4 represents severe acute and severe airflow limitation. \#Use inhalation drugs during COPD stabilization.

Our study not only compared the risk ratio of recurrence between the two groups of patients, but also compared the relative risk of annual acute exacerbation rate between the two groups. Our results are more valuable for clinical application and scientific research. Our research method is based on the natural change law of severe aggravation of COPD and the method innovation of cohort study method, which provides a new research idea for the long-term benefit of COPD hospitalization. In addition, inhalation medication in the long-term stable period has been relatively standardized at this stage. All the patients in our study regularly used ICS, before and after discharge with bacterial infection during hospitalization, all of them used antibiotics, excluded the confounding factors of non-inhaling glucocorticoids in the stable period, excluded the confounding factors of non-antibiotic treatment during hospitalization, and the results were more reliable. Moreover, a meta-analysis in 2014 found that oral hormone can significantly reduce the rate of readmission risk than the control group in obstructive lung disease. ${ }^{20}$ Our study found that the use of glucocorticoids can effectively reduce the risk of severe acute exacerbation of chronic obstructive pulmonary disease patients, the results of our study were consistent with the results of metaanalysis. The different is, the former had a shorter follow-up period and was designed to study the risk of readmission and recurrence rate of COPD, aiming at a single event of the first COPD attack, while our study is mainly aimed at the rate of future COPD exacerbations of patients in the two groups within one year, which is the frequency of the event attack over a period of time.

We are surprised to find that patients with eosinophil < 100 cells/ul use glucocorticoid can still get good long-term benefits, which seems to be contrary to the fact that most studies have found that the long-term benefits of glucocorticoid use in patients with eosinophil $<100$ th $/ \mu 1$ are not 


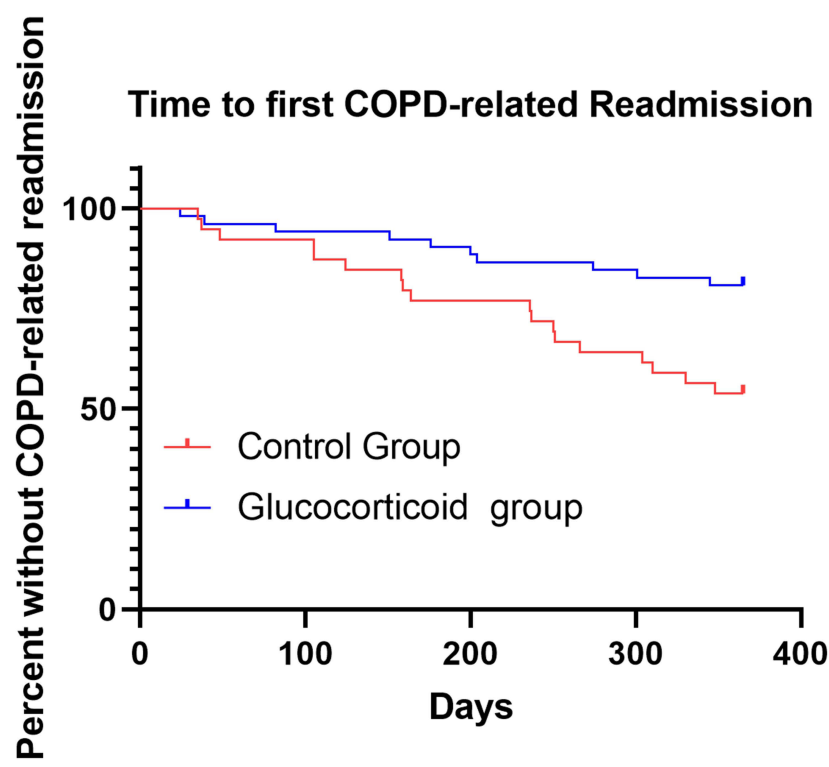

Figure 8 The time before the first COPD readmission of the patients in the control group ( $n=39$, red line) and the glucocorticoid group $(n=52$, blue line), and the HR was obtained after correcting the baseline CRP value. The median time of the first severe deterioration in the two groups was not obtained.

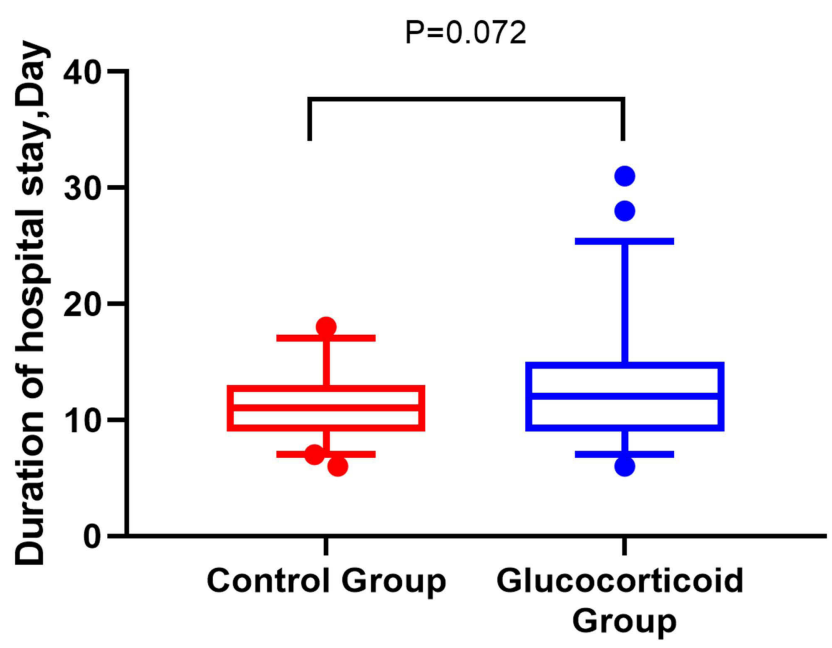

Figure 9 The hospitalization time of the glucocorticoid group and the control group, the median of the box chart is the median, and the bar stands for IQR.

significant. Eosinophil count is an important potential marker for evaluating the efficacy of steroids in the treatment of chronic obstructive pulmonary disease. A large number of clinical studies on the use of eosinophil-guided glucocorticoids have focused on long-term low-dose inhalation guidelines for stable periods. ${ }^{21}$ The 2018 Global chronic obstructive Pulmonary Disease Initiative (GOLD) guidelines recommend the use of peripheral blood eosinophil count (PBEC) to guide the selection of inhaled steroids to prevent COPD deterioration, strongly recommended when eosinophils $>300$ cells/ul, and

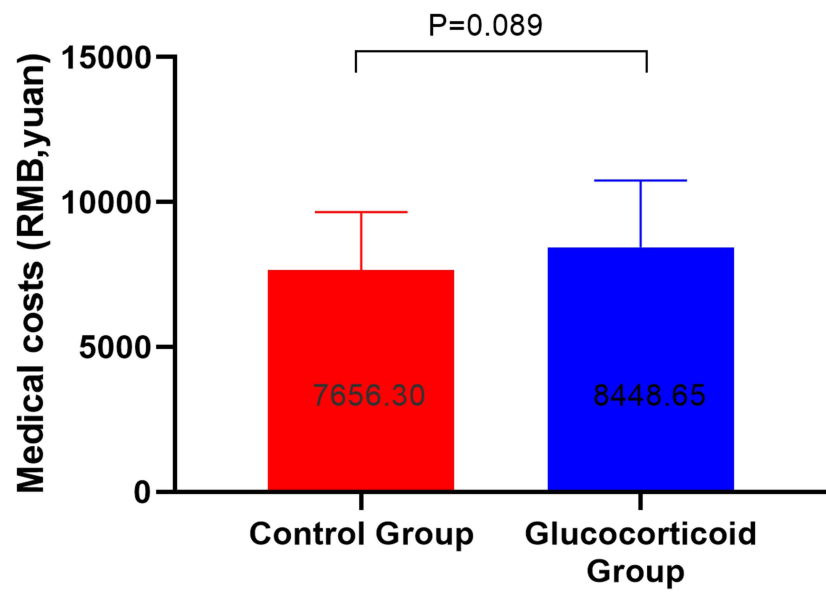

Figure 10 Medical expenses during hospitalization between the glucocorticoid group and the control group (RMB, yuan).

considered for 100-300/ul. ${ }^{8}$ Therefore, sometimes clinical medical staff may subconsciously use the rules guided by eosinophil as the rules for glucocorticoid selection in acute exacerbation, but the research population and intervention methods of the two are not consistent, so the results obtained are not necessarily similar. Our study population is in the acute episode of COPD, and the intervention is the short-term use of large doses of systemic or local glucocorticoids, which is different from the previous clinical research population and intervention methods guided by eosinophils, so our results do not violate the guidelines. In another study, The treatment failure rate was $66 \%$ in patients with a blood eosinophil count $\geq 2 \%$ who did not receive prednisolone and $11 \%$ in those who did (mean difference 55\% (95\% CI 38-73\%), p<0.001). In patients with a blood eosinophil count $<2 \%$ there was no difference in treatment failure rate. ${ }^{9}$ This suggests that patients with high levels of eosinophils treated with prednisolone have a lower risk of COPD recurrence, while patients with low eosinophils have a higher risk of COPD recurrence. ${ }^{22}$ However, it seems that our result was contrary to the study. However, the primary outcome of former study was different from our study, which aim at a single event of the first COPD attack, our study is mainly aimed at the rate of future COPD exacerbations of patients in the two groups within one year, which is the frequency of the event attack over a period of time. In addition our study was a retrospective cohort study with a single center and small sample. The overall eosinophil count in patients with a response greater than or equal to 200 cells/ul was only $15(16 \%)$. The accuracy of long-term benefits of glucocorticoid use in AECOPD patients with a response greater 
Table 2 Adverse Events

\begin{tabular}{|l|l|l|l|l|}
\hline Event & Total (N 9= 9I) & Control Group (n= 39) & Glucocorticoid Group (n= 52) & $P$ \\
\hline Any adverse event & $17(18.5)$ & $7(17.9)$ & $10(19.2)$ & 0.877 \\
\hline Any serious adverse event & $0(0)$ & $0(0)$ & $0(0)$ & \\
\hline Most frequent adverse events & & & & \\
Hypertension & $0(0)$ & $0(0)$ & $0(0)$ & $1(1.9)$ \\
Hyperglycemia & $3(3.3)$ & $2(5.1)$ & $8(15.4)$ & 0.397 \\
Gastrointestinal reaction & $13(14.3)$ & $5(12.8)$ & $2(3.8)$ & 0.729 \\
Osteoporosis & $2(2.2)$ & $0(0)$ & 0.216 \\
\hline
\end{tabular}

than or equal to $200 / \mu 1$ was limited. In this study, our results emphasize that the use of blood eosinophils as biomarker in patients with stable COPD may not be applicable to the selection of glucocorticoids in patients with acute exacerbation of COPD, and the hormonal methods of eosinophils in patients with acute exacerbation of COPD need to be further studied.

In this study, interaction analysis showed that COPD patients use glucocorticoid during hospitalization may get more long-term clinical benefits, who over 65 years old, male, normal BMI, no smoking, concomitant symptoms, GOLD3-4 grade, stable use of ICS/LABA and $\mathrm{PO}_{2} \geq$ $60 \mathrm{mmhg}$. GOLD2018 guidelines suggest that the risk factors for COPD are age, sex, airway hyperresponsiveness, infection, chronic bronchitis and so on. ${ }^{8}$ This is why we use gender, concomitant complications and GOLD grading as variables for interactive grouping. A study of 50,991 people found that they smoked 20 packs. Age or above and body mass index $<18.5 \mathrm{~kg} / \mathrm{m}^{2}$ were also risk factors for COPD, so BMI and smoking were also included in the analysis variables. $^{23}$ In addition, the therapeutic drugs during the stable period are also variables that affect the severe deterioration of COPD in patients, and PO2, which we found in the bivariate correlation, is also one of the variables that affect the severe deterioration. In this study, almost all the factors that may affect the severe deterioration of patients were included in the interaction analysis, and the biological significance of each group of variables was clearly defined in the cross-grouping diagram. This study analyzed in detail the long-term benefits of glucocorticoid therapy under various variables, and found for the first time a number of factors affecting the long-term benefits of glucocorticoid therapy in patients with AECOPD, in order to provide a reference for the evaluation of optimal glucocorticoid selection in patients with AECOPD.

\section{Advantage}

Our study found for the first time that the use of systemic glucocorticoids during hospitalization can effectively reduce rate of future COPD exacerbations in patients, and found a number of factors affecting the long-term benefits of glucocorticoids, which provides a clinical basis and evaluation direction for the selection of glucocorticoids in acute exacerbation, and is of great clinical significance. Our study found for the first time that eosinophil count as a biological index for the selection of glucocorticoids during hospitalization is not necessarily reliable, breaking the conventional inertia and opening up a new field of vision for the future study of biological indicators in stable and acute stages of chronic diseases. Based on the natural history characteristics of acute exacerbation of COPD and the theory of clinical methodology of cohort study, we break the traditional inherent mode of studying the effect of hospitalization medication on prognosis, and construct a new system for studying hospitalization treatment and long-term prognosis of the disease, which provides new research ideas for hospitalization medication and long-term effect in the future.

\section{Limitation}

Our study is a single-center retrospective cohort study, which can not achieve randomized double-blind, and may produce regional and subjective treatment bias, which can be verified by a multicenter randomized double-blind prospective study with a larger sample size in the future. In addition, due to the lack of objective and unified evaluation criteria for acute exacerbation of COPD hospitalization, it may have an impact on the results of the study. Therefore, the follow-up research direction can focus on the research and development of convenient and enforceable COPD hospitalization score criteria. Secondly, although all hospitalized patients were treated with 


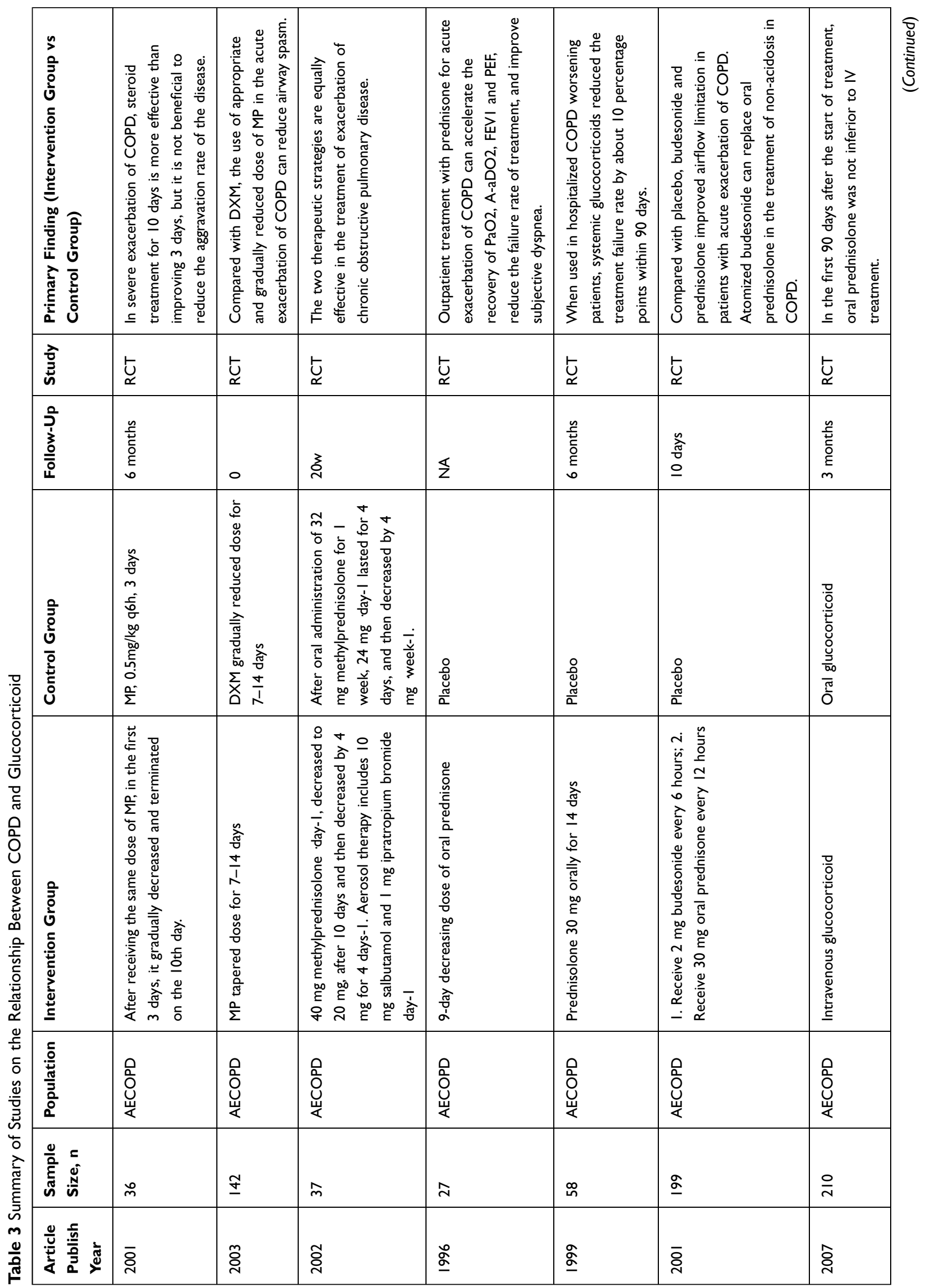




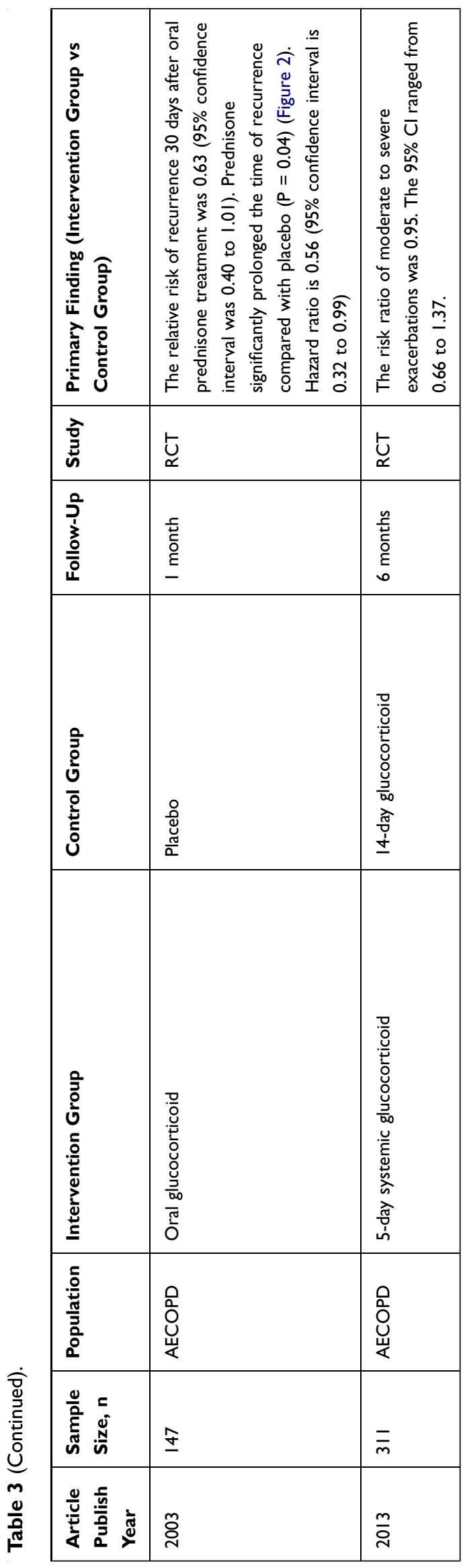

antibiotics during the first hospitalization, which reduced the antibiotic bias, we did not collect information on antibiotic treatment after re-admission, which may affect the follow-up results. Finally, as the follow-up is a retrospective review of patients after one year, it is difficult to collect the daily routine use of COPD during the stable period, which will affect the final conclusion. Future prospective studies can introduce electronic inhalation dosimeters and daily monitoring of patients' daily medication to accurately control confusion.

\section{Conclusion}

Systemic glucocorticoid use during hospitalization can effectively reduce the severe deterioration rate of COPD in patients. The number of blood eosinophils before admission, age, sex, BMI, smoking, complications, GOLD grade and stable medication may affect the longterm effect of glucocorticoids during hospitalization.

\section{Data Sharing Statement}

I declare to share all data, including all the original data for this study. The data of this study requires applicants to actively contact the corresponding author to request openness. The data will be open within 7 working days after the corresponding author agrees.

\section{Acknowledgments}

The authors acknowledge the help of Guangfa Wang, Huawei Li and Hanqiong Zhang. Guangfa Wang gave guidance to the research design of this paper. Huawei Li and Hanqiong Zhang contributed to the ethical approval process.

\section{Disclosure}

The authors report no conflicts of interest in this work.

\section{References}

1. Wang $\mathrm{C}, \mathrm{Xu} \mathrm{J}$, Yang $\mathrm{L}$, et al. Prevalence and risk factors of chronic obstructive pulmonary disease in china (the china pulmonary Health. Lancet. 2018;391(10131):1706-1717. doi:10.1016/S0140-6736(18) 30841-9

2. Chapman KR, Mannino DM, Soriano JB, et al. Epidemiology and costs of chronic obstructive pulmonary disease. Eur Respir J. 2006;27(1):188-207. doi:10.1183/09031936.06.00024505

3. WHO. World Health Report. Geneva: World Health Organization; 2000. Available from: http://www.who.int/whr/2000/en/statistics. htm:2000. Accessed August 18, 2021.

4. Mathers CD, Loncar D. Projections of global mortality and burden of disease from 2002 to 2030. PLoS Med. 2006;3(11):e442. doi:10.1371/ journal.pmed.0030442 
5. Spencer S, Calverley PM, Burge PS, Jones PW. Impact of preventing exacerbations on deterioration of health status in COPD. Eur Respir J. 2004;23:698-702. doi:10.1183/090319 36.04.00121404

6. Calverley PM, Stockley RA, Seenmungal TA, et al. Reported pneumonia in patients with COPD: findings from the INSPIRE Study. Chest. 2011;139:505-512. doi:10.1378/chest.09-2992

7. Walters JA, Wang W, Morley C, Soltani A, Wood-Baker R. Different durations of corticosteroid therapy for exacerbations of chronic obstructive pulmonary disease. Cochrane Database Syst Rev. 2011;5(10):CD006897.

8. Global Initiative for Chronic Obstructive Lung Disease (GOLD). Global Strategy for the Diagnosis, Management, and Prevention of Chronic Obstructive Pulmonary Disease (2018 REPORT). Available from: http://www.goldcopd.org. Accessed August 18, 2021.

9. Sayner A, Aytemur ZA, Cirit M, Nsal P. Systemic glucocorticoids in severe exacerbations of COPD. Chest. 2001;119(3):726-730. doi:10.1378/chest.119.3.726

10. Huiping LI, He G, Chu H, et al. A step-wise application of methylprednisolone versus dexamethasone in the treatment of acute exacerbations of COPD. Respirology. 2003;8(2):199-204. doi:10.1046/ j.1440-1843.2003.00468.x

11. Willaert W, Daenen M, Bomans P, et al. What is the optimal treatment strategy for chronic obstructive pulmonary disease exacerbations? Eur Respir J. 2002;19(5):928. doi:10.1183/090319 36.02.00268702

12. Thompson WH, Nielson CP, Carvalho P, et al. Controlled trial of oral prednisone in outpatients with acute COPD exacerbation. $\mathrm{Am} J$ Respir Crit Care Med. 1996;154(2):407. doi:10.1164/ajrccm.1 54.2.8756814

13. Niewoehner DE, Marcia L, Erbland MD, et al. Effect of systemic glucocorticoids on exacerbations of chronic obstructive pulmonary disease. $N$ Engl J Med. 1999;340(25):1941-1947. doi:10.1056/ NEJM199906243402502
14. Maltais F, Ostinelli J, Bourbeau J, et al. Comparison of nebulized budesonide and oral prednisolone with placebo in the treatment of acute exacerbations of chronic obstructive pulmonary disease: a randomized controlled trial. Am J Respir Crit Care Med. 2002;165 (5):698-703. doi:10.1164/ajrccm.165.5.2109093

15. Ynze P, Uil SM, Grotjohan HP, et al. Oral or IV prednisolone in the treatment of COPD exacerbations. Chest. 2007;132:1741-1747.

16. Aaron SD, Katherine L, Vandemheen N, et al. Outpatient oral prednisone after emergency treatment of chronic obstructive pulmonary disease. $N$ Engl J Med. 2003;348:2618-2625. doi:10.1056/NEJMoa023161

17. Leuppi JD, Schuetz P, Bingisser R, et al. Short-term vs conventional glucocorticoid therapy in acute exacerbations of chronic obstructive pulmonary disease: the REDUCE randomized clinical trial. JAMA. 2013;309(21):2223-2231. doi:10.1001/jama.2013.5023

18. Erbland ML, Deupree RH, Niewoehner DE. Systemic Corticosteroids in Chronic Obstructive Pulmonary Disease Exacerbations (SCCOPE): rationale and design of an equivalence trial. Veterans Administration Cooperative Trials SCCOPE Study Group. Control Clin Trials. 1998;19 (4):404-417. doi:10.1016/s0197-2456(98)00011-7

19. Suissa S, Dell'Aniello S, Ernst P. Long-term natural history of chronic obstructive pulmonary disease: severe exacerbations and mortality. Thorax. 2012;67(11):957-963. doi:10.1136/thoraxjnl-2011-201518

20. Walters JA, Tan DJ, White CJ. Systemic corticosteroids for acute exacerbations of chronic obstructive pulmonary disease. Cochrane Database Syst Rev. 2014;9. doi:10.1002/14651858.CD001288.pub4

21. Janson C. Treatment with inhaled corticosteroids in chronic obstructive pulmonary disease. $J$ Thorac Dis. 2020;12(4):1561-1569. doi: $10.21037 /$ jtd.2020.02.51

22. Bafadhel M, Davies L, Calverley PM. Blood eosinophil guided prednisolone therapy for exacerbations of COPD: a further analysis. Eur Respir J. 2014;44(3):789-791. doi:10.1183/09031936.00062614

23. Fang L, Gao P, Bao H, et al. Chronic obstructive pulmonary disease in China: a nationwide prevalence study. Lancet Respir Med. 2018;6:421-430. doi:10.1016/S2213-2600(18)30103-6

\section{Publish your work in this journal}

The International Journal of COPD is an international, peer-reviewed journal of therapeutics and pharmacology focusing on concise rapid reporting of clinical studies and reviews in COPD. Special focus is given to the pathophysiological processes underlying the disease, intervention programs, patient focused education, and self management protocols. This journal is indexed on PubMed Central, MedLine and CAS. The manuscript management system is completely online and includes a very quick and fair peer-review system, which is all easy to use. Visit http://www.dovepress.com/testimonials.php to read real quotes from published authors. 